REVISTA DE DERECHO UNED, NÚM. 17, 2015

\title{
HACER POSIBLE LA PAZ
}

\author{
MAKING PEACE POSSIBLE
}

\author{
Pablo Romo Cedano
}

Licenciado en Filosofía. Maestro en Teología. Candidato al Doctorado en Derechos Humanos por la UNED

Resumen: Hacer la paz posible es un tema permanente en la historia de la humanidad. Vivir en armonía, en una sociedad libre de violencia es un anhelo inalcanzado hasta ahora por la humanidad, por más que ha habido grandes y serios esfuerzos por que la paz sea posible. La paz es término que ha vivido en correlato, en un binomio permanente con el de la guerra. La paz, no es la ausencia de guerra y no es una realidad que gana terreno en tanto la guerra o el conflicto lo pierde. La paz, como concepto, trasciende la guerra y se ubica en sociedades «sin guerra», pero en constante conflicto. Pero, ¿es posible intervenir para hacer la paz posible? ¿Cómo se interviene en los últimos años para hacerla vigente?

Abstract: Making peace possible is a permanent theme in mankind history. Live in harmony, in a society without violence, is up to now an unreached aim for humanity, even though there have been great and serious efforts for making peace possible. «Peace» is a word that has lived correlated, in a permanent binomial with the word "war». "Peace» is not the absence of "war», and is not a reality that gains territory as a result of losing a war or a conflict. Peace, as a concept, transcends war and it is located in societies "without war», but in constant conflict. But, is it possible to intervene to make peace possible? How do you intervene nowadays to make it current?

Palabras clave: Paz; historia de la paz; construcción de paz.

Keywords: Peace; peace history; buiding peace. 
Recepción original: 17/08/2015

Aceptación original: 2/09/2015

Sumario: Introducción. 1. El concepto de paz. 1.a. ¿Por qué la guerra y no la paz? 1.b. Por el camino de la guerra. 2. Ante el conflicto la paz. 2.a. El conflicto social. 2.a.1. Tipos de conflictos. 2.a.2. Aproximaciones al conflicto social en la sociología clásica. 2.a.3. Siete categorías del conflicto. 2.b. Abordarlo para superarlo. 2.b.1. Las actitudes. 2.b.2. Las escuelas.

\section{INTRODUCCIÓN}

Hacer la paz posible es un tema permanente en la historia de la humanidad. Vivir en armonía, en una sociedad libre de violencia es un anhelo inalcanzado hasta ahora por la humanidad, por más que ha habido grandes y serios esfuerzos por que la paz sea posible. La paz es término que ha vivido en correlato, en un binomio permanente con el de la guerra. La paz, no es la ausencia de guerra y no es una realidad que gana terreno en tanto la guerra o el conflicto lo pierde. La paz, como concepto, trasciende la guerra y se ubica en sociedades «sin guerra», pero en constante conflicto.

El fin de la guera fría, ha colocado a la paz en un terreno ventajoso para crecer con independencia de las grandes guerras catastróficas que arrinconaban al concepto a «ceses al fuego», "pactos de no agresión» o efímeras "treguas». Cierto que aún hay muchas expresiones de guerra tradicionales que no acaban y pareciera que se acentúan en algunas regiones del planeta. Pero a pesar de éstas decenas de guerras registradas en los años noventas y en el inicio del dos mil; la paz incorporó en su espacio semántico la necesidad de reivindicar todos y cada uno de los derechos humanos y planetarios reconocidos y en reconocimiento, como condición sine quan non la paz no es posible.

Así, la paz se ubica como necesidad también en donde no se cumplen cabalmente los derechos humanos y donde el planeta se destruye por la devastación y la contaminación. La paz se requiere en sociedades con democracias débiles donde los conflictos sociales emergen con reivindicaciones de nuevas relaciones, nuevas distribuciones de los bienes naturales o nuevos reconocimientos a identidades ancestrales o emergentes.

Pero, ¿es posible intervenir para hacer la paz posible? ¿Cómo se interviene en los últimos años para hacerla vigente? 
Nuestro texto atraviesa por el concepto de paz, hace una aproximación al de conflicto social y recoge lo que los académicos y activistas de la paz dicen sobre el tema. Concluimos con lo que estamos trabajando en nuestra tesis doctoral, si es posible contruir la paz, si es posible intervenir deliveradamente para hacerla posible y también es posible intervenir en los procesos para exsacervar la guerra, la violencia y la no-conciliación. La paz es posible y tiene que tener adjetivos que califiquen y cualifiquen.

\section{EL CONCEPTO DE PAZ}

Para iniciar considero que es indispensable hacer una aproximación al término de Paz a fin de responder a la pregunta por hacer posible la paz. Preguntémonos ¿Qué es la paz?, ¿la paz tiene siempre un correlato permanente de no-paz que le llamamos guerra, o más bien es la guerra la que existe y la paz es simplemente la ausencia de guerra? ¿La paz y la guerra se auto-implican? ¿Son un binomio indispensable para la existencia de la humanidad? ¿Pueden existir o una o la otra por sí mismas? En el caso que así fuera, ¿es posible hacer posible la paz?

Ciertamente son innumerables las formas y los tipos de paz de los que podemos extraer noticias de la historia, y no menos innumerables los criterios con base en los cuales ha sido intentada por varios autores su clasificación. Pero responder a tales preguntas considero que es indispensable hacer un recorrido por la historia de la filosofía, y particularmente por la filosofía política, sin detenerme en ella en cuanto tal, sino en cuanto lo que implica para la búsqueda que emprendemos y seguir algunos autores que nos ayuden a esclarecer nuestra pregunta por la paz.

El concepto de paz, afirma Norberto Bobbio, está tan vinculado al de guerra que los dos términos, paz y guerra, constituyen el ejemplo típico de antítesis, como los análogos orden-desorden, concordia-discordia. Y de tal manera están ligados que uno depende del otro:

Si de los dos términos de una pareja uno de ellos siempre es el fuerte o independiente, y el otro el débil o dependiente, ello radica en el hecho de que las dos situaciones señaladas por los dos términos no son existencialmente relevantes de la misma manera. (...) El término fuerte es el primero (guerra) ${ }^{1}$.

${ }^{1}$ BOBBIO, Norberto. «Paz» en FERNÁNDEZ SANTILLÁN, José Norberto Bobbio: El filósofo y la Política. Antología, México, FCE, 2. ${ }^{a}$ ed. 2002, pág. 312.

(C) UNED. Revista de Derecho UNED, núm. 17, 2015 
¿Es esto cierto? ¿Se auto-implican y se requieren uno al otro? Iniciemos nuestro recorrido.

\section{1.a. ¿Por qué la guerra y no la paz?}

Norberto Bobbio (1909-2004), en varios de sus ensayos compilados por Fernández Santillán, hace un recorrido histórico desde Tucídides (460 a C-396 a C) hasta prácticamente nuestros días (el fin de la guerra fría) y en este recorrido hace una primera constatación: «Una historia sin narraciones de guerra, no sería la historia de la humani$\mathrm{dad}^{2}$ ». Esta trágica afirmación pone muy en entre dicho nuestra búsqueda de paz y la garantía que debería hacer el derecho a ella.

En la historia de la filosofía, dice Bobbio, «en diversas ocasiones se ha señalado que siempre ha existido una filosofía de la guerra, en tanto que es mucho más reciente la filosofía de la paz, de la que el primer gran ejemplo es $\mathrm{Kant}^{3}{ }^{3}$. Desgraciadamente es muy cierto, pues ya los griegos antiguos habían desarrollado con bastante claridad una filosofía de la guerra, por llamarla como lo hace el propio Bobbio. Los relatos y pasajes de los antiguos filósofos son por decir lo menos desesperanzadores.

La filosofía política ha sido una continua reflexión en torno al problema de la guerra. Las preguntas que se han hecho los pensadores más destacados ligados al gobierno, al buen gobierno, y la civilidad siempre coinciden en cuestionar la guerra como el único modo de establecer la paz. En la medida que las guerras han sido devastadoras cada vez más, en capacidad y en extensión, se preguntan los políticos y pensadores en la manera de reducir su impacto, abreviar su existencia y estudiar la manera de crear pactos que normen su realización. ¿Cuáles son las causas que conducen a la guerra? ¿Es un camino inexorable de la humanidad? ¿Es la única manera de hacer la historia? ¿Así se camina fatalmente hacia el futuro? ¿Existen remedios? ¿Las guerras son el único modo para generar evolución en la humanidad?

Si caminamos en esta reflexión podemos constatar con Bobbio y con muchos otros que el tema de la paz, o, lo que es lo mismo, del orden (interno), siempre ha sido tratado por derivación con respecto al tema de la guerra o del desorden: la paz como desembocadura, una

\footnotetext{
${ }^{2}$ BOBBIO, Norberto. «Paz» ibid. pág. 313.

${ }^{3}$ Ibid. Pág. 324.
} 
de las posibles desembocaduras, de la guerra (el orden, como derivación de la revolución). Hagamos un simple recorrido histórico.

Erasmo de Rotterdan ${ }^{4}$ (1466-1536) en el siglo XV empuja a un pacifismo muy agudo, reacción de los conflictos entre protestantes y católicos, y plantea que es indispensable responder al por qué de la guerra y no a la inversa. Es decir, exige una razón o justificación para la guerra, de lo contrario «no es justa». Erasmo anima esta reflexión incómoda para el Imperio Español en expansión y deja toda una escuela con Tomás Moro (1478-1535) o Guillaume Budé (1467-1540) y en España a Juan de Vergara (1492-1557) y los hermanos Alfonso (1500-1532) y Juan de Valdés (1509-1541) y después los frailes dominicos de Salamanca, Fray Domingo de Soto (1494-1570), Fray Francisco de Vitoria (1486-1546) y la propia escuela de Salamanca ${ }^{5}$. Con Erasmo y los subsiguientes pensadores se abre una discusión importante en preguntarse por la paz y plantearla como una idea posible, por más que se convierta en el lugar sin lugar. De esta búsqueda humanista tendremos un nuevo imaginario y nuevo horizonte: existe la posibilidad de que la humanidad conviva en la paz, sin necesidad de guerras. De ahí que muchos se den a la tarea de hacerla realidad. Bartolomé de las Casas, Vasco de Quiroga, Antonio de Montesinos y tantos más. La utopía era un horizonte que se abrió en la nueva tierra, en el «nuevo mundo» de América. Desde entonces, con más fuerza, la paz se convirtió en una exigencia y un ideal.

Años después, el gran pensador francés, Francois-Marie Arouet Voltaire (1694-1778), en su Dictionnaire philosophique exige una explicación por la guerra y no por la paz, es decir, considera natural que la paz sea y no la guerra. De esta manera debate contra Montesquieu (1689-1755) sobre el tema y claramente quiere responder a Nicolás Maquiavelo (1469-1527) con su obra El Príncipe. Voltaire dedica a la guerra un buen artículo en su diccionario y si bien es largo vale la pena tomarlo como otro punto de partida para nuestra reflexión.

Define la guerra como:

El hambre, la peste y la guerra son los más terribles azotes de la humanidad. Los dos primeros nos vienen de la Providencia, pero la guerra nos viene de la imaginación de trescientas o cuatrocientas personas esparcidas por toda la faz de la tierra bajo el nombre de prínci-

${ }^{4}$ Erasmo en su Querela pacis, Cf. http://www.thelatinlibrary.com/erasmus/querela. shtml

${ }^{5}$ La literatura es muy abundante. Por citar dos clásicos: PINTO DE OLIVEIRA, Carlos Josaphat. Las Casas Todos os directos para todos, Sao Paulo, Ed. Loyola, 2000. PARISH, Helen-Rand y WEIDMAN, Harold E. Las Casas en México. Historia y obra desconocidas, México, FCE, 1996. 
pes o ministros; quizá por esta razón en muchas dedicatorias se les llama imágenes vivas de la Divinidad. (...)

Lo maravilloso de esta empresa infernal es que cada jefe de los asesinos hace bendecir sus banderas e invoca a Dios solemnemente antes de ir a exterminar a su prójimo. Cuando un jefe sólo tiene la suerte de degollar a dos o tres mil hombres, no da gracias a Dios, pero cuando consigue despachar diez mil y destruir alguna ciudad, entonces manda entonar un canto de acción de gracias, compuesto en lengua desconocida para todos los que pelearon y lleno de barbarismos. El mismo canto sirve para celebrar los matrimonios, los nacimientos y los homicidios. (...)

Si vuestro vecino llega a ser demasiado poderoso durante la paz, ¿quién os impide serlo tanto como él? Si él contrajo alianzas, vosotros podéis contraerlas también. Si tiene pocos religiosos, en cambio tiene muchos manufactureros y soldados. Imitad su buen ejemplo. Si instruye mejor a sus marinos, instruid mejor a los vuestros; todo esto es muy justo. Pero exponer al pueblo a la más horrible miseria con la idea, tan quimérica a menudo, de destruir a vuestro querido hermano el serenísimo príncipe vecino vuestro, semejante consejo no es digno del presidente honorario de una compañía pacífica ${ }^{6}$.

Sin embargo, la gran filosofía de la historia, de la edad moderna incipiente, que se mueve del iluminismo al positivismo, del historicismo al marxismo, y llega a nuestro siglo con Spengler ${ }^{7}$ y con Toynbee ${ }^{8}$, y a nuestros días con una de las últimas obras de Karl T. Jaspers ${ }^{9}$ (1883-1969), se hace la pregunta sobre ¿cuál es el significado de la guerra, de una guerra cada vez más destructiva y siempre menos comprensible en sus fines y efectos?

Entonces, ¿cómo seguir nuestro análisis del concepto de paz, ahora en la modernidad? ¿Es un correlato de guerra? ¿Camina sola? Bobbio nos orienta para un abordaje en su propio ensayo $\mathrm{Paz}^{10}$, acercándose a los términos con los siguientes capítulos: Paz negativa y positiva; La paz como valor; La idea de paz perpetua; Pacifismo institucional y pacifismo ético; El equilibrio del terror, y el Tercero para la paz.

${ }^{6}$ VOLTAIRE. Diccionario Filosófico, Buenos Aires-Miami, Ed. Libro, dot, 2006.

${ }^{7}$ Oswald SPENGLER (1880-1936), especialista en filosofía de la historia, estableció una teoría cíclica sobre el desarrollo de las civilizaciones.

${ }^{8}$ Según Arnold J. TOYNBEE (1889-1975), las civilizaciones no son sino el resultado de la respuesta de un grupo humano a los desafíos que sufre, ya sean naturales o sociales. De acuerdo con esta teoría, una civilización crece y prospera cuando su respuesta a un desafío no sólo tiene éxito, sino que estimula una nueva serie de desafíos; una civilización decae como resultado de su impotencia para enfrentarse con los desafíos que se le presentan.

${ }_{9}$ JASPERS, Karl. El origen y meta de la historia, Barcelona, Ed. Altaza, 1995 (Vom Ursprung und Ziel der Geschichte, de 1949).

${ }^{10}$ BOBBIO, N. Op. cit. págs. 311-342. 
Quizá sea conveniente, seguir algunas de sus huellas y avanzar mejor en el concepto con él y con la propia historia del pensamiento.

\section{1.b. Por el camino de la guerra}

Bobbio parte de la constatación que hemos también nosotros hecho arriba, de que los dos términos de la pareja guerra - paz, el fuerte es la guerra y el débil es la paz. Y que hasta ahora en nuestro recorrido histórico podemos definir la paz sólo definiendo la guerra.

La guerra la entiende el turinés en este primer momento «cuando dos o más grupos políticos se encuentran entre sí en una relación de conflicto cuya solución es dejada al uso de la violencia (...) se tiene una situación de conflicto cada vez que las necesidades o los interese de un individuo o un grupo son incompatibles con los de otro individuo o grupo y por tanto no pueden ser satisfechos sino en perjuicio de uno o de otro» ${ }^{11}$. A esta aproximación a la guerra podríamos agregar no sólo que «son» incompatibles, sino que se les percibe así. La guerra, así entendida, puede generarse por la disputa de bienes, territorio, intereses o valores. En el caso de la disputa por el territorio o territorialismo, será cuando existe una competencia de muchos individuos o grupos por la posesión de un bien escaso que se encuentra en el territorio del otro. La guerra, en cuanto solución de un conflicto entre grupos políticos mediante violencia, es una de las maneras de reparar una disputa, a la que generalmente se recurre cuando los medios pacíficos no han tenido efecto o se presume que no lo tendrá.

El territorialismo es una forma particular de la defensa del propio ámbito espacial en el que todo individuo está interesado. Pero no es el único pretexto. Otro nivel de guerra es por el rango, es decir, por la defensa de la preeminencia, de la jerarquía que permite a quien ocupa los niveles más altos gozar de ciertos privilegios. La lucha se da tanto por acceder a éste como por mantenerse en el privilegio.

Estas luchas se establecen cuando los acuerdos o las amenazas no funcionan. La distinción entre situaciones en las que los conflictos son resueltos habitualmente con acuerdos y situaciones en las que los conflictos son solucionados incluso por medio de la violencia corresponde a la distinción, según Bobbio, entre estado agonista, regido por reglas sustanciales y procedimentales que prevén varias formas de conflicto y las maneras de su solución pacífica (piénsese en las

${ }^{11}$ El uso de grupos políticos en lugar de Estado es deliberado, pues lo usa en términos weberianos. 
normas consuetudinarias o autorizadamente establecidas que regulan los conflictos de competencia entre los diversos órganos del Estado), y estado polémico, que, aun previendo reglas para la solución de las controversias, no excluye el recurso al uso de la fuerza, aunque esté también en algunos casos regulado por normas. Aquí incorporamos un nuevo elemento: fuerza diferenciándose de violencia. De esta manera podemos hacer una distinción entre reglamentación de los actos de fuerza y la reglamentación de los actos de fuerza usados. Más adelante abordaremos el uso del derecho para el primer caso y también encontraremos al Derecho Internacional Humanitario aquí inscrito. Se entiende fuerza, en este contexto, como el uso de medios capaces de infligir sufrimiento físico, y, en consecuencia, no incluyen ni la violencia psicológica, esta es, el uso de medios de manipulación de la voluntad ajena para obtener los efectos deseados, ni la violencia institucional o estructural, es decir la violencia que deriva de la relación de dominio dentro de ciertas instituciones, como la fábrica, la escuela, el ejército, para no hablar de las llamadas institucionales totales, como el manicomio, las cárceles, las organizaciones de grupos fanáticos religiosos o políticos, regulados por una disciplina férrea inclinada a excluir cualquier comportamiento que no esté orientada al objetivo ${ }^{12}$.

Para este contexto el concepto la violencia física es la que distingue a la guerra de otras formas de ejercicio de poder del hombre sobre el hombre y en consecuencia son expresiones metafóricas todas aquellas denominaciones de "guerra de nervios», "guerra psicológica» y otras por el estilo. Esta aproximación de violencia y guerra es acotada pero auxilia a definir los límites de éstas.

Si contrastamos los términos fuerza con violencia descubrimos que violencia tiene una connotación axiológicamente negativa que fuerza no tiene. Y de hecho, el derecho lo usa como una virtud y una expresión de un Estado de Derecho. Es decir, que hay un uso lícito y un uso ilícito de la fuerza.

Por ejemplo, la muerte cuando es producida por un asesinato es un hecho violento; cuando es generada por un verdugo, cumplimentando una orden de un juez es un acto de fuerza. Así, como se usa el término fuerza, así el de guerra, que es análogo, y que para el término guerra las reglas son las internacionales.

Entonces, el uso de la fuerza así entendida, es un medio para resolver conflictos. Pero no basta hacer referencia al uso de la fuerza

12 BOBBIO. Op. cit. pág. 316. 
entendida como violencia lícita y autorizada. La guerra siempre es, en primer lugar, una fuerza ejercida colectivamente: como tal, tradicionalmente es distinguida del duelo, que pone frente a frente a dos individuos. Y en segundo lugar para que se pueda hablar de guerra es preciso que no se trate de violencia, aun entre grupos políticos independientes, esporádica, discontinua, sin consecuencias relevantes. La violencia colectiva y no accidental de la guerra siempre presupone de alguna manera una organización, un aparato, aunque rudimentario, es lo que distingue a la guerrilla (la que es una especie de guerra) del motín aun llevado a cabo con armas.

La guerra, en consecuencia, es violencia organizada grupalmente que se prolonga por un cierto periodo de tiempo ${ }^{13}$.

Usualmente se dice que dos estados están en estado de Guerra ante una situación de conflicto armado, violento; sin embargo no usa el término estado de Paz, para definir a un Estado en tiempos de no-guerra. Dos grupos políticos se encuentran en condiciones de paz cuando entre ellos no existe conflicto a cuya solución ambos contribuyan recurriendo al ejercicio de una violencia colectiva, durable y organizada.

Sin embargo, estas primeras definiciones nos alcanzan a cubrir todo, pues puede haber situaciones de permanente conflicto sin estar en guerra, es decir el estado de paz no excluye el conflicto. Pensemos en lo que se conoció como guerra fría que era una especie de paz con permanente conflicto.

Por otro lado, la fuerza potencial es una amenaza constante de guerra que no rompe la paz. recordemos aquello de 'si quieres la paz prepárate para la guerra' (si vis pacem para bellum $)^{14}$.

Si seguimos con el vocablo paz descubrimos que $p a z$ se usa para designar la firma de acuerdos que pone fin a la guerra como por ejemplo la Paz de Westfalia y la Paz de Basilea, es decir el conjunto de acuerdos con los que dos grupos políticos cesan hostilidades, delimitan las consecuencias de la guerra y norman sus relaciones futuras. Y también se refiere a periodos de tiempo donde se viven las consecuencias de tales tratados, como el periodo de la Paz Augusta.

De igual modo, hablamos de paz cuando reina un imperio en un orden establecido, por ejemplo, la pax romana o bien la pax ameri-

\footnotetext{
${ }^{13}$ Ibid. Pág. 321.

${ }^{14}$ Cita de Vegencio en De Re Militari, Siglo IV D. C.
} 
cana ${ }^{15}$. Situaciones de no guerra posible y ordenes de gobierno dictatoriales. Nos lleva a pensar que eso es una contradicción.

Así vamos asumiendo que el termino paz se nos desliza de las manos en cuanto le ponemos ejemplos concretos. Entonces, ¿Cómo lo abordamos? ¿Debemos seguir pensando en este inquietante binomio paz-guerra y sus derivado de fuerza y violencia?

En los últimos años, Johan Galtung (1930-) amplía el término de paz no sólo estableciéndolo como ausencia de guerra (negativa) sino desde la ausencia de violencia y entonces hace una aproximación hacia la violencia personal e incluye la estructural o institucional ${ }^{16}$. Él nos ayuda ver la paz de dos formas: la negativa, que consiste en la ausencia de violencia personal, y la positiva, que consiste en la ausencia de violencia estructural. Esta segunda sólo se puede instaurar mediante un radical cambio social y que, en consecuencia, debe avanzar al parejo de la promoción de la justicia social, del desarrollo político y económico de los países subdesarrollados, con la eliminación de las desigualdades. Es decir, el amplio cumplimiento de los derechos humanos, incluyendo aquellos que le llamaremos de primera generación, con y fundamentalmente los económicos, sociales y políticos y con los de tercera generación, como son los del desarrollo y la cooperación internacional. Ya entraremos en el debate de los siguientes derechos o los conocidos como de tercera generación. Aquí lo importante es rescatar que el concepto de paz atraviesa necesariamente por el cumplimiento de una normatividad que garantice estructuralmente una convivencia humana en respeto. Vayamos tomando nota de este concepto para incorporarlo en nuestra idea de paz.

Entonces, según parece, pensar la paz es algo que va más allá que la propia guerra, y las normas que la limitan o permiten, e implica la generación de condiciones favorables de desarrollo o crecimiento de la persona humana. Si esto es así, entonces, hacer posible la paz es una tarea deliberada y no sólo un cumplimiento mecánico o bien de acuerdos, leyes o normas; o en su caso de técnicas mecanizadas. Pareciera que es más bien un arte que se les da a unos cuantos. ¿Esto es cierto? ¿No todo ciudadano puede hacer posible la paz?

${ }^{15}$ SÖLLE, Dorothee. The Arms Race Kills Even Without War, Philadelphia, Fortress Press, 1983.

${ }_{16} \mathrm{~J}$. GALTUNG ha generado toda una escuela que enfatiza la dimensión de la violencia estructural como parte de los obstáculos para la paz. Su sitio oficial es: http:// www.transcend.org/ 


\section{ANTE EL CONFLICTO LA PAZ}

Parece indispensable que iniciemos ahora este apartado por algunos de los grandes pensadores de la paz que recientemente han discutido con lo que hemos planteado hasta el momento. Dentro de los y las pensadores contemporáneos de la paz encontramos de manera destacada a Johan Galtung ${ }^{17}$, que ya hemos citado y que cambia nuestro paradigma binario de paz-guerra; John Paul Lederach, quien desde su activismo por la paz y su convicción de fe -Quáquero- evoluciona en su pensamiento liberal para escuchar las voces de los pobres de latinoamérica; Sue Williams, premio Nobel de la paz que también incorpora la visión desde la mujer como acción para la paz; Vincent Fisas ${ }^{18}$, un clásico del Estado Español que estudia conciensudamente muchos conflictos armados en el mundo; y Tania Paffenholz, mujer reflexiva que entra en escena de la discusión por hacer posible la paz desde la multiculturalidad y desde nuevas herramientas hermenéuticas.

La reflexión sistemática sobre el abordaje de los conflictos sociales y particularmente de los conflictos armados toma auge después de la segunda mitad del siglo Xx. La paz ya no está en los autores más contemporáneos asociada irremediablemente al binomio con la guerra, pero de alguna manera sí lo está al uso de la fuerza o la violencia, en el sentido que hablábamos antes. Es fundamentalmente en Estados Unidos donde se desarrollan los centros de investigación pioneros en el tema. Sin embargo, en el norte de Europa y a finales del siglo xx en España la preocupación por la paz se ve manifestada en la creación de centros de reflexión en torno a ella. En los países del sur, Costa Rica tiene mucho que decir, por ejemplo, de acuerdo a sus políticas de neutralidad, no violencia y creación de espacios para reflexionar en torno a la paz.

Por su parte, muchos países que han vivido situaciones de conflictos armados, guerras, revoluciones o conflictos sociales de alta intensidad, tienen la necesidad de reflexionar en torno a su propia vida y al proceso vivido, tratando de rescatar las lecciones aprendidas. Tales lecciones hoy se presentan como triunfos que quieren compartir con

${ }^{17}$ GALTUNG, Johan. Citado por ARENAL, Celestino. Investigación sobre la paz: pasado, presente y futuro, Congreso Internacional sobre la paz, México, Universidad Autónoma de México, Instituto Investigaciones Jurídicas 1987, pág. 571; Serching for Peace. The road to transcend. Ed. Pluto Press. London. 2000; Peace by Peaceful Means, Sage/PRIO, Oslo, 1996.

${ }^{18}$ FISAS, Vicenç. Procesos de Paz y Negociación en Conflictos Armados. Paidos Estado y Sociedad 119. Barcelona. 2004; Cultura de paz y gestión de conflictos, Icaria/UNESCO, Barcelona/París, 1998. 
otros países y pueblos para poder superar los conflictos (meta-conflictos en términos de Galtung). La reflexión y los aportes para la paz en estos países contribuye notablemente al avance de la paz en diferentes planos a los cuales parece necesario abordar. Desde África del Sur, después de su transición a la democracia y el fin del Apartheid, se han hecho muchas reflexiones en torno a prácticas de reconciliación, de justicia y de construcción de paz en muchos niveles (multi-track). Algunos países de América Latina tienen historias de violencia, guerra y conflictos internos recientes que han generado lecciones muy relevantes para la construcción de la paz. En los últimos años el ejemplo de Colombia es muy cercano y en un delicado momento. Por su parte, hay que tomar en cuenta en este sentido qué se ha hecho en el proceso de Irlanda del Norte o en el país Vasco.

¿Cómo abordar este conocimiento? Parece que hay dos grandes fuentes a los cuales acudir. Por un lado tenemos a los teóricos que están sistematizando experiencias vividas y reflexiones de paz, y por el otro experiencias en directo, testimonios que nos pueden ayudar para nuestro propósito de construcción de paz y reivindicación del derecho a la paz como ejercicio de cualquier ciudadano. Empecemos con una aproximación al conflicto.

\section{2.a. El conflicto social}

Una vez que hemos superado el binomio de paz-guerra y entendiendo los componentes que ésta tiene, los pensadores de la paz nos proponen el concepto conflicto social como espacio que requiere trascenderse para que la paz sea posible ${ }^{19}$. Acudamos a algunos maestros de la sociología para afinar nuestro concepto.

Los conflictos sociales tienen su origen en el orden y la integración social. La imagen armónica de la sociedad establecida por Rousseau, donde basa su concepto de sociedad en la idea de un sistema estable de sus elementos y el consenso de todos ellos para el mantenimiento de la sociedad, se contrapone a la que Hobbes establece que la sociedad está en constante cambio y ésta se encuentra integrada por elementos contradictorios que contribuyen al cambio social, así, la coac-

${ }^{19}$ Este apartado es deudora de una práctica de más de cinco años en la investigación sobre la conflictividad social en México con un equipo de estudiantes comprometidos en el cambio social, laborando desde la institución que hago parte: SERAPAZ. Cf. ROMO, P. «Observatorio de la Conflictividad Social en México» en Carrillo, P. Investigación-docencia interdisciplinaria, gestión del conocimiento y tecnología. UNAM, México. 2015. 
ción de algunos elementos sobre otros se debe establecer para el mantenimiento de la sociedad.

Como dice Jesús Giner ${ }^{20}$, ambas concepciones, como arquetipos de referencia, han inspirado varias teorías sociales distintas e incluso han tomado recíprocamente aspectos una de la otra. Estos enfoques sociológicos han sido retomados por numerosos autores de diversas tradiciones entre los que se encuentran a Talcott Parsons (1902-1979) con su funcionalismo estructural o C. Wright Mills (1916-1962). Siguiendo a Hobbes, el conflicto es un aspecto básico del cambio social, ya que permite resolver divergencias de grupos o colectividades para alcanzar un nuevo modelo de integración social. El estudio del cambio social ha desarrollado esquemas y teorías basadas en el conflicto social. De hecho, Oscar Lewis (1914-1970) establece que la sociología se basa en el estudio del conflicto social, mientras que Robert Redfield (1897-1957) la basa en la teoría funcionalista. Dentro de los que se inclinan por la primera opción tenemos a Stanisław Ossowski (1897-1963), Thomas Burton Bottomore (1920-1992), Alain Touraine (1925-), Anthony Giddens (1938 - ) y entre otros.

\section{2.a.1. Tipos de conflictos}

Los tipos de conflictos son múltiples y complejos de clasificar, debido a la variedad de actividad conflictiva, actores y objetivos que puede darse en cualquier situación humana que requiera una real, sentida o ficticia unificación, resolución o transformación de antagonismos. Los más frecuentes son los conflictos en el seno de las élites; conflictos populares; conflictos comunitarios y societarios; revoluciones; guerras entre países; comportamientos anímicos; conflictos privados o contra sociedades agresivas.

Según Giner la tipología empírica del conflicto nos dan dos criterios orientativos: la unidad social en la que se produce el conflicto (rol social, grupo social organizado, etc.), y la categoría de los grupos o elementos en conflicto (iguales frente a iguales, superiores frente a inferiores, etc.). Estos criterios posibilitan la elaboración de teorías particulares del conflicto, tales como la del conflicto de roles, de las relaciones internacionales, de la minoría, etcétera. Más adelante veremos la composición por sus actores, que también puede ser una manera de tipología del conflicto. De hecho, la «ley» de formación de

${ }^{20}$ GINER, Jesús. «Conflicto Social» en REYES, Román. Dir. Diccionario Crítico de Ciencia Sociales. Ed. Plaza y Valdéz. Madrid y México. 1988.

(C) UNED. Revista de Derecho UNED, núm. 17, 2015 
conflictos sociales está basada en la constitución de actores agrupados por su interés «grupos de interés». Veamos cómo nos podemos aproximar a un concepto útil para nuestro tema.

\section{2.a.2. Aproximaciones al conflicto social en la sociología clásica}

Marx y Engels estudian un tipo de conflicto social: el producido por la lucha de clase debido a la desigualdad en la propiedad de los medios de producción. Y a partir de esta aproximación de la conflictividad social van más allá estableciendo que la historia se construye por la lucha de clases: «es el principal motor de la historia que forzosamente produce el cambio de estructuras sociales» ${ }^{21}$. En la sociología de los conflictos se acepta la explicación marxista clásica de lucha de clases, al menos en lo referente a sociedades en fases iniciales de industrialización. Pero en la sociedad neocapitalista aparecen nuevos elementos sociales, nuevas formas de producción y ocupación como pueden ser los tecnócratas, el movimiento estudiantil, el feminismo, el ecologismo, los sindicatos charros $^{22}$, los movimientos indígenas, entre otros que requieren una tipología diferente o más comprehensiva. De ahí que los neo-marxistas se hayan esforzado por caracterizar conflictos sociales diferentes en la clásica lucha de clases ${ }^{23}$.

El sociólogo Lewis A. Coser (1913-2003) intenta fundir la teoría anterior del conflicto social con los aportes funcionalistas del conflicto y su aproximación la hace como «la lucha por los valores y por el status, el poder y los recursos escasos, el curso de la cual los oponentes desean neutralizar, dañar o eliminar a sus rivales». Un conflicto será «conflicto social» cuando trascienda lo individual y proceda de la propia estructura de la sociedad. El autor intentó elaborar una teoría general de las funciones integradoras del conflicto social, en la que afirma que ciertas formas de conflicto son necesarias para el mantenimiento de la identidad, de la cohesión y de la delimitación de un grupo social, hasta tal punto que en ocasiones un determinado grupo

${ }^{21}$ MARX, K. El 18 Brumario de Luis Bonaparte. Introducción. Alianza Editorial. España. 2003.

${ }^{22}$ Los Sindicatos Charros, son expresiones corporativas del sistema mexicano. Se copian del corporativismo de tiempos del fascismo italiano y se adecuan a la realidad mexicana. Es la clase obrera incorporada al sistema, como estructura de control. La mayor parte de los sindicatos en el país son parte de este sistema corporativo que militan en el Partido Revolucionario Institucional, partido en el poder desde 1929 con una interrupción de dos sexenios (2000-2012).

${ }^{23}$ En este sentido vale la pena revisar a POULANTZAS, Nicos. Poder Político y clases sociales en el estado capitalista. Siglo XXI, México 1969. 
social no existiría si no viviera en conflicto o en preparación para un conflicto inminente. El intento de integración a partir de una situación de desigualdad social provoca conflicto, pero dicho conflicto es precisamente el factor del cambio social. Esta aproximación nos puede ayudar en nuestra tarea entendiendo la necesidad del conflicto social para superar situaciones de marginación, concomitantes o diferentes a la clase social ${ }^{24}$.

Otro autor que puede ayudarnos es Ralf Dahrendorf ${ }^{25}$ quien ha intentado reformular la teoría de los conflictos sociales, particularmente la marxista, elaborando un modelo teórico capaz de explicar la formulación de grupos de conflicto y su acción social para lograr la integración mediante los necesarios cambios de estructura en la sociedad. Retomando a Marx, Dahrendorf establece que los conflictos oponen siempre a dos únicos grupos con intereses totalmente contrarios: el que define los intereses establecidos y el que no los acepta y pugna el cambio de estructuras sociales.

Los factores estructurales del cambio social son de dos tipos según Marx: los exógenos que actúan sobre el sistema desde fuera y los endógenos que surgen del propio sistema por su peculiar estructura y su funcionamiento. Marx afirmó que el propio sistema social origina fuerzas endógenas que lo transforman, como la lucha de clases, producto de las contradicciones internas de la sociedad capitalista. La crítica de Dahrendorf es que Marx ha reducido todos los conflictos sociales a conflictos de clase. Para Marx la lucha de clases desemboca en la revolución necesariamente, mientras que para Dahrendorf señala que la sociedad es más dinámica y los sistemas no necesariamente se perpetúan en la revolución permanente, sino en mutaciones internas. El caso más frecuente de solución o transformación de un conflicto social, según Dahrendorf es el que la clase dominante adopta las nuevas ideas y se auto-transforma de manera adecuada para desactivar los posibles factores de una revolución. No necesariamente todas las contradicciones sociales llevan a una revolución.

Otro elemento importante en la discusión de Dahrendorf está en la propiedad privada. Si para Marx la desaparición de la propiedad privada estaba en estrecha relación con el fin de la lucha de clases, para Dahrendorf la evolución del capitalismo ha demostrado que la propiedad de medios de producción y clase social pueden ir disociados, y que lo que determina el conflicto de clases no es la propiedad

\footnotetext{
${ }^{24}$ COSER, L. Las funciones del conflicto social. Ed. FCE, México. 1961.

${ }^{25}$ DAHRENDORF, R. Sociedad y Libertad, Madrid, Tecnos. 1966.
} 
sino el control de los medios de producción en manos de tecnócratas y burócratas sin propiedad alguna.

De esta manera el conflicto social tiene otras claves que se traducen en poder. Generalmente el conflicto gira en torno al poder, pero dicho poder se ejerce para establecer el control sobre bienes y servicios (salarios, recursos naturales, producción, mejora de las condiciones de trabajo, menor discriminación social, etcétera). El poder toma formas muy sutiles que escapan a esquemas claramente coactivos y se manifiestan en aspectos tales como la manipulación ideológica o la discriminación lingüística, étnica, sexual o religiosa. No obstante, poder social y poder económico tienen grandes nexos de unión y se auto-implican. Nuevamente, para Dahrendorf la clave estructural del conflicto social es el poder que se basa en la desigual distribución de la autoridad entre personas y grupos de la sociedad. La autoridad existe en toda colectividad y forma parte de su organización, de modo que siempre existirán relaciones de dominación en la sociedad; desde las burdas estructuras totalitarias a las más refinadas de la democracia.

A veces se piensa que el conflicto plantea la disyuntiva por el poder: quien lo tiene lucha por mantener la autoridad y el que no la tiene por conseguirla. Sin embargo no siempre es así. Las relaciones de dominación son más complejas y a menudo se intenta modificar el orden establecido desde la propia autoridad para conseguir la permanencia del estatus quo.

Otro elemento que hay que tener en cuenta en todo conflicto social es el tiempo (cronos). Los tiempos del conflicto social varían y dependen de los momentos, pero podemos con claridad verificar que hay dos grandes etapas: la revolución, como cambios rápidos y violentos y los procesos de transformación o evolución progresiva. Los actores sociales en conflicto constantemente se debaten al interior de su auto-comprensión en el dilema estratégico o táctico de sus acciones en función del «tiempo del conflicto».

Por otro lado, la importancia del conflicto se puede analizar por dos características mensurables en escalas diferentes: la intensidad y la violencia. La intensidad viene determinada por el grupo de participación y la violencia se refiere a las formas de expresión de los conflictos (huelgas, negociación, amenazas, guerra, etc.). La intensidad y la violencia decrecen, según Dahrendorf, en la medida en que los grupos de interés tienen posibilidades de organizarse, en que los conflictos existentes en una sociedad no confluyen sino que permanecen disociados, y en la posibilidad de movilidad social en la estructura de 
clases sociales. Así pareciera que la amplitud de los cambios de estructuras depende más de la intensidad del conflicto que de su violencia (una mayor intensidad provoca un cambio mas radical), sin embrago, para muchos actores sociales en conflicto la inminencia del cambio depende de la violencia (a mayor violencia del conflicto el cambio será más inmediato).

Existen tres actitudes básicas frente a los conflictos sociales: la represión, típica de sociedades totalitarias, que deviene ineficaz e incluso peligrosa en su intento de hacer desaparecer toda oposición; la pseudo-regulación fundada en la represión sutil, la reacción mas corriente y la regulación efectiva que intenta canalizar los conflictos según pacto o procedimiento aceptado por las partes en conflicto.

\section{2.a.3. Siete categorías del conflicto}

Parece que para nuestra aproximación es conveniente adoptar una definición de conflicto social compleja que recoja lo anterior, nos ayude a caracterizar el conflicto y que sea lo suficientemente englobante que incluya nuestra temática.

Ya mencionamos algunos de los elementos a tomar en cuenta, que nos aporta Coser y Dahrendorf, veamos ahora otros dos autores contemporáneos, que conjugan muchos de estos elementos y elaboran una definición que quizá nos resulte útil para nuestro tema. Empecemos con el investigador catalán Vincenc Fisas:

El conflicto es un proceso interactivo que se da en un contexto determinado. Es una construcción social, una creación humana, diferenciada de la violencia (puede haber conflictos sin violencia, aunque no violencia sin conflicto), que puede ser positivo o negativo según cómo se aborde y termine, con posibilidades de ser conducido, transformado y superado (puede convertirse en paz) por las mismas partes, con o sin ayuda de terceros, que afecta a las actitudes y comportamientos de las partes, en el que como resultado se dan disputas, suele ser producto de un antagonismos o una incompatibilidad (inicial, pero superable) entre dos o más partes, y que expresa una insatisfacción o desacuerdo sobre cosas diversas ${ }^{26}$.

Fisas, director de la Escola de Cultura de Pau de la Universitat Autónoma de Barcelona, en su aproximación al conflicto social nos presenta 8 elementos o componentes de un conflicto social. Él reconoce que es un proceso (1), es decir, no es un evento estático, muta,

${ }^{26}$ FISAS, Viçens. Cultura de Paz y Gestión de paz. Barcelona/París, Icaria/ UNESCO, 1998. 
cambia diacrónicamente; se presenta en un momento histórico concreto (2); es una construcción social (3), no es fortuito o fruto de una voluntad supra humana que fatalmente determina la historia; no necesariamente se expresa con violencia (4). El conflicto social puede abordarse hacia un horizonte de construcción de los actores o a la destrucción de alguno de ellos (5). Se puede abordar y conducir, transformar y superar (6). Participan actores sociales en conflicto que mutan en el tiempo (7). El objeto del conflicto es diverso (8).

En tanto, el Dr. Pedro Luis Lorenzo Cadarso nos propone una definición que contiene siete elementos:

Un proceso de interacción contenciosa entre actores sociales que comparten orientaciones cognitivas, movilizados con diversos grados de organización y que actúan colectivamente de acuerdo con expectativas de mejora, de defensa de la situación preexistente o proponiendo un contraproyecto social ${ }^{27}$.

Veamos de una por una las características o elementos del Conflicto Social.

\section{a. Proceso}

Proceso: "un conflicto no debe confundirse con los actos multitudinarios a que dé lugar, puesto que éstos se inscriben en una sucesión lógico-causal de hechos históricos de la que forman parte, sin que su espectacularidad implique, necesariamente, que tengan una especial trascendencia de cara a la explicación global del conflicto ${ }^{28} 》$ Esto implica que no toda movilización es un conflicto, las acciones visibilizan el conflicto, pero no lo contienen y que las acciones visibles son las expresiones del conflicto, pero no el conflicto en sí, por si solas.

\section{b. Interacción contenciosa}

La Interacción contenciosa significa que «el conflicto debe caracterizarse por la lucha abierta entre grupos sociales opuestos. Dicha confrontación tendrá un nivel ideológico-cultural, caracterizado por la valoración que se haga de la situación preexistente y, por extensión, de las expectativas de cada grupo, y otro nivel de acción social, concretado en

${ }^{27}$ LORENZO CADARSO, Pedro Luis. Fundamentos teóricos del Conflicto Social. Editorial Siglo XXI. España Ed. 2001, pág. 12.

${ }^{28}$ LORENZO. Op cit., pág. 15. 
los repertorios tácticos empleados, en las formas de acción colectiva, y en la tipología general de los conflictos sociales.»

Esto significa que es importante distinguir entre discurso y acción colectiva, en base a los intereses que están de por medio. Lucha abierta entre dos o más actores. El conflicto ocurre cuando existe una confrontación clara entre grupos sociales opuestos. La confrontación se da en tres niveles o en alguno de los tres: 1) ideológico-cultural, 2) a nivel de expectativas de los actores confrontados y 3 ) en las formas de acción colectiva.

\section{c. Actores sociales}

Los Actores sociales son "grupos asociativos o comunitarios, con las autoridades públicas usualmente presentes, bien como víctimas de la acción colectiva, bien como responsables, cuando menos, del orden público.» Significa que los actores sociales en conflicto se agrupan en torno al objetivo común y que de alguna manera la autoridad pública está presente, no necesariamente como contraparte directa o principal.

La ley de formación de conflicto social es la existencia de «grupos de interés». Hay que distinguir entre el «cuasi-grupo» que es una categoría social o subgrupo que comparte ciertos intereses derivados de una situación común de sus elementos (estudiantes, vecinos, comunidades, etc.), y el "grupo de interés» que posee una organización, un programa de acción y unos intereses muy definidos (sindicatos, partidos políticos, movimientos sociales). El cuasi-grupo puede evolucionar hasta convertirse en grupo de interés, que es el desencadenante del conflicto social, al concretar las razones de las contradicciones y radicalizar la acción de los subgrupos.

\section{d. Movilización}

La Movilización «los actos y las críticas deben ser conscientes mentalmente, racionales en términos estratégicos y coordinados socialmente. También hace referencia a las formas y niveles de compromiso de los actores sociales con el movimiento social: cuadros dirigentes, militantes, grupos de apoyo, simpatizantes, etc.; y, por último, a las estructuras de movilización de recursos que emplee el grupo. Así, las acciones colectivas que emprenden los actores en conflicto no son espontáneas porque existe un nivel de compromiso y de organización en ellas; es 
decir, porque están encaminadas a un objetivo (forman parte de un proceso como lo hemos definido).

Subrayamos que la movilización colectiva está organizada y motivada por un interés y aparece cuando los actores sociales vislumbran la posibilidad política de lograr dicho interés. Así mismo, la posibilidad política para el logro de un interés dado, determina el tipo de movilización. Entendemos por movilización al conjunto de acciones públicas que realizan los actores.

Actos conscientes mentalmente, racionales en términos estratégicos y coordinados socialmente. Formas y niveles de compromiso de los ASC con el movimiento social (dirigentes, militantes, grupos de apoyo, simpatizantes, etc.).

\section{e. Orientaciones cognitivas}

Las Orientaciones cognitivas significa que "el grupo ha de compartir ideas y objetivos, una similar valoración de la situación y unas expectativas asimilables, se conceptualicen éstas como ideologías, cultura o marcos interpretativos. También hace referencia a las llamadas identidades colectivas, esto es, al autorreconocimiento como grupo y a la existencia de lazos de sociabilidad y solidarios en su seno».

La formación de un grupo implica que sus integrantes tienen común idea ante el conflicto y la identidad colectiva puede generarse someramente mientras el conflicto perdure.

\section{f. Organización}

La Organización expresa el "grado de institucionalización de las entidades que gestionan la movilización, ya sean preexistentes o generadas por el propio movimiento. También a los tipos concretos de movimientos sociales organizados: partidos politicos, sindicatos, organizaciones de bases, grupos de presión, etc.; y, por último, al liderazgo que se establezca en su seno.»

Podríamos definir varios niveles de organización, por ejemplo, un nivel "preexistente», otro nivel «ad hoc», etc. Al distinguir niveles de organización tendríamos la posibilidad de captar los cambios en el tipo de organización según el momento del conflicto y su evolución a movimiento social, en su caso. 


\section{g. Objetivos}

Por último, Lorenzo Cadarso presenta a los objetivos del movimiento: «su dimensión táctica-objetivos moderados, pragmáticos, radicales, maximalistas, utópicos, etc.- como a su relación con procesos sociales más amplios: objetivos revolucionarios, reformistas y conservadores.»

Si pudiéramos combinar por sus elementos las definiciones de estos autores, lograríamos una definición con nueve elementos básicos de lo que es un conflicto social que nos será útil para nuestro caso en estudio. Es decir, habrá que agregar a la definición de Cadarso dos elementos que son el reconocimiento de que el conflicto social es un producto de la misma sociedad y que se pueden abordar deliberadamente de alguna manera.

\section{2.b. Abordarlo para superarlo}

Una vez que hemos hecho una aproximación al término del conflicto social, es importante preguntarse cómo es posible abordarlos para cambiarlos, como nos dice Fisas, y solucionarlos o transformarlos en su caso y en el fondo, hacer posible la paz.

Hagamos un recorrido por las actitudes ante el conflicto y los modos como históricamente se han abordado los conflictos este recorrido nos permitirá constatar que en efecto, los conflictos son abordables y las intervenciones son variadas, tanto por los actores como los terceros en discordia.

\section{2.b.1. Las actitudes}

El mismo Vincent Fisas nos dice que «el proyecto de construir una cultura de paz en buena medida no es otra cosa que el reto planetario de abordar los conflictos desde otra mirada, con otros utensilios y con otros propósitos». De esta manera lo primero que hay que hacer para abordar un conflicto social, como cualquier otro conflicto es reconocerlo como tal. No reconocerlo implica devaluar no sólo el conflicto sino también al actor, al oponente. Con frecuencia, esta devaluación pública del nivel real conflictivo va acompaña de una negativa a reconocer la entidad del oponente, en un intento de evitar interferencias o presiones exteriores que podrían derivar hacia una negociación cara a cara. La negación de la existencia del conflicto social, es un acto reflejo de los regímenes autoritarios. Mientras más autoritario es un 
sistema, la negación es recurrente, pues no puede -no tiene capacidad- de aceptar la contestación, la diferencia o la disidencia. En cambio, mientras más incluyente y democrático es un sistema, recurre menos a la negación, pues está familiarizado con lo diferente, con opiniones y posturas contrarias y tiene mayor capacidad de abordar y procesar el conflicto. Así, la negación no es el camino.

Según Fisas frente al conflicto, sea cual sea su naturaleza, hay una multiplicidad de posibilidades de reacción, tanto a nivel individual como colectivo, dándose las siguientes actitudes, según se acepte, evite o niegue:

- Superación. Se reconoce su existencia y hay voluntad de superarlo -ventaja- se reconoce su existencia y se procura sacar provecho del mismo;

- Negación. Se evita reconocer su existencia;

- Evasión. Se reconoce su existencia, pero sin deseos de enfrentarse a él;

- Acomodación. Se reconoce su existencia, pero se opta por no darle respuesta alguna;

- Arrogancia. Se reconoce su existencia, pero sin darle una respuesta adecuada;

- Agresividad. Se combate con una respuesta hostil, violenta y/o militar.

El autor no pretende juzga con actitudes morales de personas, se trata de actitudes colectivas caracterizadas ante el conflicto. Son respuestas de los actores ante el conflicto (hasta la no respuesta o la negación).

De esta manera, veremos a los actores sociales en conflicto, a las partes beligerantes comportarse a lo largo del proceso de maneras diversas: no se trata de una actitud permanente a lo largo del conflicto; por el contrario, van interactuando en su contexto y asumiendo actitudes de acuerdo a sus posibilidades de «avanzar» o los riesgos de «retroceder».

\section{2.b.2. Las escuelas}

Por otra parte, veamos a los que estudian las intervenciones en los conflictos. ¿Cómo se interviene? ¿Cuáles han sido las escuelas de paz que intervienen en conflictos. 
La historia de las intervenciones en los conflictos en orden a «construir paz» es muy reciente y lo hacen con la convicción de que sí es posible intervenir en ellos. La manera de hacerlo ha ido cambiando con los años. Intervenir un conflicto es irrumpir en una dinámica propia del conflicto desde una perspectiva diferente de las partes beligerantes con un propósito deliberado. En este caso, para construir la paz, hacerla posible.

Los teóricos sobre las intervenciones para construir la paz han ido aumentando en los últimos años. Los primeros investigadores e interventores de conflictos datan de mediados de la segunda guerra mundial y sus reflexiones se centran en la manera de «resolver» conflictos.

Lederach $^{29}$ en sus reflexiones, aún las más recientes, ubica tres grandes escuelas en la intervención de conflictos, a saber:

- Resolución de conflictos. Este concepto indica la necesidad de entender cómo el conflicto empieza y termina, y busca una convergencia de los intereses de los actores.

- Gestión de conflictos. Es un concepto que reconoce que el conflicto no puede resolverse en el sentido de librarse de él, y que pone el acento en limitar las consecuencias destructivas del conflicto. Es un concepto que no recoge el sentido amplio de pacificación, y se limita a los aspectos técnicos y prácticos del esfuerzo. Intenta realinear las divergencias.

- Transformación de conflictos. El acento en la naturaleza dialéctica del conflicto. El conflicto social es un fenómeno de la creación humana que forma parte natural de las relaciones humanas.

Las tres escuelas, que presenta Lederach van sucediéndose en el tiempo. Los primeros investigadores y peacemakers pretenderán «resolver» los conflictos. En un segundo momento, la reflexión y su práctica se centra en la manera de cómo "gestionar» los mismos. Y en un tercer momento, ya a finales del siglo xx la reflexión y la práctica van a llevar a abordar los conflictos para «transformarlos». Lederach en su proceso personal y con su equipo de investigadores llega a la conclusión que los conflictos no se «resuelven», se «transforman». Este salto epistémico lo realiza en sus prácticas y discusiones con colegas constructores de paz, según él mismo confiesa, en El Salvador en el contexto de las pláticas de paz a fines de los 80’s.

${ }^{29}$ LEDERACH, J. P. Construyendo la paz. Gernika. 1998. Capítulo 1, págs. 25-35. 
En tanto, la investigadora Thania Paddenholz clasifica en cinco las grandes escuelas para el abordaje de los conflictos:

The mainstream peacebuilding literature usually comes up with three schools (management, resolution and transformation), but I would like to add two more, one for historical reasons (complementary school) and one due the new discussions that currently take place in research (alternative discourse school) that could have implications on practitioners' work30.

Ella desde su centro de Investigación es Ginebra, realiza una clasificación en cinco momentos, o generaciones -diré yo- de constructores de paz.

\section{Primera generación: Manejo de conflictos}

La escuela que hace una aproximación al conflicto como un «manejo o gestión» es la escuela más vieja en el trabajo y construcción de paz. Está muy ligada a los trabajos de por la paz ya institucionalizados en las leyes internacionales. Los constructores de paz según esta escuela enfocan su acción en aquellos que tienen el poder y que tienen capacidad para desarrollar a gran escala violencia o bien para frenarla mediante negociación con ellos ${ }^{31}$. Según Paffenholz la contribución mayor de esta escuela radica en su enfoque atendiendo a los actores de alta intensidad. Sin embargo ha sido fuertemente criticada esta escuela porque soslaya los actores intermedios y los menores. Ignora la acción de facilitación de los propios proceso internos durante las negociaciones y no asume las causas que generan la conflictividad.

\section{Segunda generación: La resolución de conflictos}

La escuela de Resolución de Conflictos se enfoca en las causas de la conflictividad y reconstruye las relaciones entre las partes. Esta escuela, fundamentalmente se desarrolla en Europa y Estados Unidos en los espacios académicos ensayando sus prácticas en talleres con actores no oficiales cercanos a las partes en conflicto ${ }^{32}$. Sus seguidores se encuentran en actores de sociedad civil y líderes de comunidades.

${ }^{30}$ PAFENHOLZ, Thania. «Understanding peacebuilding theory: Management, resolution and transformation.» En Rev New Routes 2/2009, pág. 3.

${ }^{31}$ Son los casos recientes de Sudán y Aceh Cf. Miall et al. 1999, 158-168; Paffenholz 2001. a , 75-81; Richmond 2005, 89-96.

${ }^{32}$ FISHER, R. Interactive Conflict Resolution. In «Peacemaking in International Conflict: Methods and Techniques», ed. Zartman/Rasmussen. Washington, D. C. Uni- 
Ha sido criticada esta escuela por los seguidores de la primera generación porque consideran que la intervención que realizan no incide necesariamente en los actores de primer nivel (los señores de la guerra) y por tanto no se logran acuerdos de largo aliento ${ }^{33}$. Con el tiempo también fueron criticados porque los niveles alcanzados de su intervención no eran suficientes para los conflictos de mayor nivel ${ }^{34}$.

\section{Tercera generación: la complementación de las anteriores}

La tercera generación de constructores de paz intenta hacer una síntesis entre las dos generaciones anteriores tomando lo bueno y recuperando las lecciones de la insuficiencia de alcance. A mediados de los años 90 esta generación de constructores de paz -peacebildersempiezan a discutir sobre esta síntesis.

Paffenholz nos dirá que hay tres grandes aproximaciones a esta síntesis. La realizada primera por Fisher y Keashly que la llama «Contingency model for third party intervention in amed conflicts» ${ }^{35}$. La segunda estará representada por Bercovitch y Rubin con un modelo semejante al de Fisher y Kaeshly. Y la tercera aproximación a este ejercicio sintético esta representado por la diplomacia multi track con Diamond y McDonald ${ }^{36}$.

\section{Cuarta generación: La transformación de conflictos}

Esta escuela está enfocada a la transformación desde las causas más profundas de la conflictividad armada. Esta basada en diferentes comprensiones de la construcción de la paz.

ted States Institute of Peace Press. 1997. Y. KELMAN, H. Informal Mediation by the Scholar/Practitioner. In «Mediation in International Relations: Multiple Approaches to Conflict Management», ed. Bercovitch/Rubin. London: Macmillan. 1992.

${ }_{33}$ BERCOVITCH, J., RUBIN, B. 1992. Mediation in International Relations. Multiple Approaches to Conflict Management. London. St. Martin's Press. 1984.

${ }^{34}$ RICHMOND, O. 2001. Rethinking Conflict Resolution: The Linkage Problematic Between 'Track I' and 'Track II'. The Journal of Conflict Studies Fall '01. 2001.

${ }^{35}$ FISHER \& KEASHLY, L. "The potential complementarity of mediation and consultation within a contingency model of third party intervention.» En Journal of Peace Research, 1991. 28: 29-42.

${ }^{36}$ DIAMOND, L., J. MCDONALD. Multi- Track Diplomacy, A Systems Approach to Peace, West Hartford, CT: Kumarian Press. 1996. 
Los representantes de esta generación son Rupesinghe, Lederech en su segundo momento de reflexión sobre la paz ${ }^{37}$. La propuesta esta basada en la construcción de una «estructura de paz de largo plazo». Esta estructura estaría soportada por los potenciales de reconciliación de la sociedad. Ledererach ve, en coincidencia con la escuela de Resolución de Conflictos, la necesidad de reconstruir las relaciones destruidas, enfocándose en la reconciliación con las capacidades que tiene la sociedad en ese sentido.

Un tercer aspecto de esta escuela está centrado en ayudar a los actores internos con intervenciones externas.

\section{Quinta generación: El discurso alternativo}

La quinta escuela, o quinta generación que Paffenholz llama «alternativa» es una escuela emergente que surge de los escritos de Featherstone (2000); Richmond (2005); MacGinty (2006) y Heathershaw (2008). Se trata de de construir las prácticas internaciones evidenciando que el discurso de los peacebuilders ha devenido «self-referential»-autoreferencial-, desconectado de las necesidades reales de la gente.

La escuela Alternativa no presenta una teoría completa o compleja que abarque todo. Presenta algunos puntos y focaliza las necesidades desde la perspectiva de las necesidades cotidianas de la «gente».

Esta centrada en una visión desde «el sur» enfatizando que los peacebuilders contienen en su acción una inherente énfasis conservador legitimando las soluciones modernas, el estatus quo y al mercado (Bendaña 2003). La escuela Alternativa tiene que ver con una crítica post-hegemónica al poder y a la sociedad opresora y excluyente. Critica la acción occidental de decir «cómo intervenir en conflictos» y enfatiza en la necesidad de equilibrar el diálogo asimétrico.

Según Paffenholz la contribución mayor de esta escuela es la relevancia del enfoque que establece en la gente ordinaria, en las voces desdibujadas y en los énfasis críticos a la modernidad y la perspectiva occidental.

${ }^{37}$ LEDERACH, J. P. cuenta cómo fue su cambio de pensamiento en Latinoamérica conversando y trabajando en la guerra de El Salvador. Cf. Conferencia en la Universidad de Cartagena, Colombia, el 27 de febrero de 2014, Video: https://www.youtube.com/watch?v=4gZKF-Pyiv4 consultado el 5 de abril de 2015 . 
Síntesis de las generaciones

De las cinco generaciones la autora establece que las conclusiones a las que arriba es que aún no hay un concepto de intervención en los conflictos sociales cabal y que es necesario hacer una seria reflexión teórica sobre el «hacer paz» ${ }^{38}$ :

All of these theories already have an impact on realities. Knowing where one's underlying theories come from is therefore a first step in a critical practitioner's reflection on peace work ${ }^{39}$.

Las cinco generaciones nos muestran la deliverada intención de hacer la paz posible. Cada una en su momento, con sus debilidades y límites han tenido el firme propósito de no dejar que crezca el conflicto, sino intervenir desde un enfoque diferente y pararlo. La paz es algo deliverado y planeado y no es consecuencia de la ausencia de guerra o conflicto.

\section{A Modo de Primeras Conclusiones}

Habiendo llegado a afirmar que es posible hacer la paz y no solamente conformarnos como una paz posible, la intervención en las guerras y en los conflictos desde una visión diferente a la de los beligerantes puede hacer posible una paz, cada vez más aproximada a los ideales que representa el cabal cumplimiento de los derechos humanos todos y beneficiando a todas y todos. Quizá no se resuelva el conflicto del todo; no sólo se manejen las expresiones de violencia, pero sí se pueda llegar a un primero y después a un segundo y tercer estadio de relación transformada paulatinamente: en una especie de aproximación asintótica hacia los estándares de paz que se van haciendo día a día en las instancias internacionales como la UNESCO o las Declaraciones que han seguido a la de Luarca ${ }^{40}$. Todo ello considerando como prioridad las víctimas, los pobres, las mujeres, las poblaciones excluidas acestralmente de palabra y protagonismo en la construcción de las nuevas relaciones.

38 «Doing peace work».

${ }^{39}$ PAFENHOLZ, Thania. "Understanding peacebuilding theory: Management, resolution and transformation.» En Rev New Routes 2/2009, pág. 5.

${ }^{40} \mathrm{http}: / /$ www.ugr.es/ revpaz/documentacion/rpc_n1_2008_doc1.pdf consultado el 10 de julio 2015. 


\section{BIBLIOGRAFÍA}

BOBBIO, Norberto. «Paz» en FERNÁNDEZ SANTILLÁN, José Norberto Bobbio: El filósofo y la Política. Antología, México, FCE, 2. ${ }^{a}$ ed. 2002.

BERCOVITCH, J., RUBIN, B. 1992. Mediation in International Relations. Multiple Approaches to Conflict Management. London. St. Martin's Press. 1984.

COSER, L. Las funciones del conflicto social. Ed. FCE, México. 1961.

DAHRENDORF, R. Sociedad y Libertad, Madrid, Tecnos. 1966.

DIAMOND, L., J. MCDONALD. Multi- Track Diplomacy, A Systems Approach to Peace, West Hartford, CT: Kumarian Press. 1996.

FISAS, Viçens. Cultura de Paz y Gestión de paz. Barcelona/París, Icaria/UNESCO. 1998.

- Procesos de Paz y Negociación en Conflictos Armados. Paidos Estado y Sociedad 119. Barcelona. 2004; Cultura de paz y gestión de conflictos, Icaria/UNESCO, Barcelona/París. 1998.

FISHER, R. Interactive Conflict Resolution. In «Peacemaking in International Conflict: Methods and Techniques», ed. Zartman/Rasmussen. Washington, D. C. United States Institute of Peace Press. 1997.

GALTUNG, Johan. Citado por ARENAL, Celestino. Investigación sobre la paz: pasado, presente y futuro, Congreso Internacional sobre la paz, México, Universidad Autónoma de México, Instituto Investigaciones Jurídicas 1987,

- Serching for Peace. The road to transcend. Ed. Pluto Press. London. 2000;

- Peace by Peaceful Means, Sage/PRIO, Oslo. 1996.

JASPERS, Karl. El origen y meta de la historia, Barcelona, Ed. Altaza. 1995.

KELMAN, H. Informal Mediation by the Scholar/Practitioner. In «Mediation in International Relations: Multiple Approaches to Conflict Management», ed. Bercovitch/Rubin. London: Macmillan. 1992.

LEDERACH, J. P. Construyendo la paz. Reconciliación sostenible en sociedades divididas. Bilbao. Gernika. 1998. Capítulo 1.

MARX, K. El 18 Brumario de Luis Bonaparte. Alianza Editorial. España. 2003. 
PARISH, Helen-Rand y WEIDMAN, Harold E. Las Casas en México. Historia y obra desconocidas, México, FCE. 1996.

PINTO DE OLIVEIRA, Carlos Josaphat. Las Casas Todos os directos para todos, Sao Paulo, Ed. Loyola. 2000.

ROMO, P. «Observatorio de la Conflictividad Social en México» en Carrillo, P. Investigación-docencia interdisciplinaria, gestión del conocimiento y tecnología. UNAM, México. 2015.

SÖLLE, Dorothee. The Arms Race Kills Even Without War, Philadelphia, Fortress Press. 1983.

LORENZO CADARSO, Pedro Luis. Fundamentos teóricos del Conflicto Social. Editorial Siglo XXI. España Ed. 2001.

\section{Revistas}

FISHER, R. \& KEASHLY, L. "The potential complementarity of mediation and consultation within a contingency model of third party intervention.» En Journal of Peace Research. 1991. 28: 29-42.

GINER, Jesús. «Conflicto Social» en REYES, Román. Dir. Diccionario Crítico de Ciencia Sociales. Ed. Plaza y Valdéz. Madrid y México. 1988.

PAFENHOLZ, Thania. «Understanding peacebuilding theory: Management, resolution and transformation.» En Rev New Routes 2/2009.

RICHMOND, O. 2001. Rethinking Conflict Resolution: The Linkage Problematic Between 'Track I' and 'Track II'. The Journal of Conflict Studies Fall '01. FISHER. 1997.

VOLTAIRE. Diccionario Filosófico, Buenos Aires-Miami. Ed. Libro, dot. 2006.

\section{Fuentes en la Internet}

https://www.youtube.com/watch?v=4gZKF-Pyiv4 consultado el 5 de abril de 2015.

Erasmo de Róterdam, en su Querela pacis, Cf. http://www.thelatinlibrary.com/erasmus/querela.shtml

http://www.ugr.es/ revpaz/documentacion/rpc_n1_2008_doc1.pdf consultado el 10 de julio 2015.

https://www.transcend.org consultado el 1. ${ }^{\circ}$ de julio 2015.

(C) UNED. Revista de Derecho UNED, núm. 17, 2015 
